University of Nebraska - Lincoln

DigitalCommons@University of Nebraska - Lincoln

Ralph Skomski Publications

Research Papers in Physics and Astronomy

November 1998

\title{
Unidirectional anisotropy in ultrathin transition-metal films
}

\author{
Ralph Skomski \\ University of Nebraska-Lincoln, rskomski2@unl.edu \\ H.-P. Oepen \\ Max-Planck-Institut für Mikrostrukturphysik \\ J. Kirschner \\ Max-Planck-Institut für Mikrostrukturphysik
}

Follow this and additional works at: https://digitalcommons.unl.edu/physicsskomski

Part of the Physics Commons

Skomski, Ralph; Oepen, H.-P.; and Kirschner, J., "Unidirectional anisotropy in ultrathin transition-metal films " (1998). Ralph Skomski Publications. 18.

https://digitalcommons.unl.edu/physicsskomski/18

This Article is brought to you for free and open access by the Research Papers in Physics and Astronomy at DigitalCommons@University of Nebraska - Lincoln. It has been accepted for inclusion in Ralph Skomski Publications by an authorized administrator of DigitalCommons@University of Nebraska - Lincoln. 


\title{
Unidirectional anisotropy in ultrathin transition-metal films
}

\author{
R. Skomski,* H.-P. Oepen, and J. Kirschner \\ Max-Planck-Institut für Mikrostrukturphysik, Weinberg 2, D-06120 Halle, Germany
}

(Received 5 December 1997)

\begin{abstract}
Unidirectional magnetic anisotropies in low-symmetry magnetic thin films such as cobalt on vicinal copper surfaces are investigated. Possible explanations of the observed uniaxial anisotropies are competing anisotropy (CA) coefficients and Dzyaloshinskii-Moriya (DM) interactions. Unidirectional CA is an interesting mechanism occurring in low-symmetry magnets and involves neither antiferromagnetic exchange nor spin canting. It is visible in the easy-cone regime and decides, for example, whether the preferential magnetization direction points up or down a stepped surface. In the case of $\mathrm{Co} / \mathrm{Cu}(11 n)$ films, however, the anisotropy direction speaks in favor of DM-type interactions. [S0163-1829(98)03541-3]
\end{abstract}

\section{INTRODUCTION}

The phenomenon of magnetic anisotropy and its atomic explanation is one of the most compelling subjects in solidstate and surface science. Magnetic anisotropy means that the energy of a magnet contains an anisotropic contribution $E_{a}(\mathbf{M})=E_{a}(-\mathbf{M})$, where $\mathbf{M}$ is the magnetization. ${ }^{1,2}$ In most cases, this anisotropy gives rise to symmetric hysteresis loops $M(-H)=-M(H)$, where $H$ is the external field and $M$ is the average magnetization in the field direction (dashed line in Fig. 1). A unidirectional shift of the hysteresis loop (solid line in Fig. 1) is observed, for example, in exchangeanisotropic magnets such as cobalt particles coated by cobaltous oxide CoO.,4 A similar effect is caused by Dzyaloshinskii-Moriya (DM) interactions, ${ }^{5-7}$ which occur in metallic spin-glasses and in low-symmetry insulators such as $\alpha-\mathrm{Fe}_{2} \mathrm{O}_{3} \cdot{ }^{8-10}$ In the context of ultrathin-film magnetism, unidirectional Kerr hysteresis loops have been observed for stepped Co surfaces: $\mathrm{Co} / \mathrm{Cu}(1117)$ films vicinal to fcc (001) exhibit an unambiguous but unexplained unidirectional shift of order $1 \mathrm{mT} .^{11,12}$ Although nonferromagnetic spin structures cannot be ruled out in low-symmetry $3 d$ films, ${ }^{13}$ it is not possible to ascribe the observed loops to the well-known exchange between ferromagnetic and antiferromagnetic phases as in $\mathrm{Co} / \mathrm{CoO}$.

The aim of this paper is to draw attention to the phenomenon of unidirectional anisotropies in low-symmetry transition-metal films and to discuss possible physical explanations. In particular, we will discuss under which conditions competing anisotropy (CA) contributions yield unidirectional hysteresis loops.

\section{COMPETING ANISOTROPIES}

Consider, for the moment, uniformly magnetized films characterized by the magnetization direction $\mathbf{n}=\cos \theta \mathbf{e}_{z}$ $+\sin \theta \cos \phi \mathbf{e}_{x}+\sin \theta \sin \phi \mathbf{e}_{y}$, where it is common to write the magnetic anisotropy energy in terms of expressions such as $^{14-16}$

$$
\begin{aligned}
E_{a}(\theta)= & K_{1} \sin ^{2} \theta+K_{1}^{\prime} \sin ^{2} \theta \cos \left(2 \phi-2 \phi_{0}\right) \\
& +K_{2} \sin ^{4} \theta+\cdots .
\end{aligned}
$$

Here $K_{1}$ and $K_{2}$ are the first and second uniaxial anisotropy constants, respectively, and $K_{1}^{\prime}$ describes deviations from the uniaxial anisotropy. The disadvantage of the functions established by Eq. (1) is that they are neither complete nor orthogonal. The nonorthogonality means, for example, that $K_{1}$ is not a true lowest-order anisotropy constant but contains an admixture of higher-order atomic contributions. ${ }^{17,18}$ In fact, the validity of Eq. (1) is limited to highly symmetric structures, such as low-indexed bcc and fcc surfaces.

A complete and orthonormal set of functions is obtained by using Legendre polynomials. ${ }^{17,18}$ For example, neglecting higher-order and nonuniaxial contributions we reproduce the well-known expression

$$
E_{u}=\frac{\kappa_{2}}{2}\left(3 \cos ^{2} \theta-1\right)+\frac{\kappa_{4}}{8}\left(35 \cos ^{4} \theta-30 \cos ^{2} \theta+3\right),
$$

where the $\kappa_{n}$ 's are the $n$th order uniaxial anisotropy coefficients. Minimizing Eq. (2) with respect to $\theta$ yields the phase diagram Fig. 2(b). Note that the uniaxial relations $K_{1}$ $=-3 \kappa_{2} / 2-5 \kappa_{4}$ and $K_{2}=35 \kappa_{4} / 8$ transform Fig. 2(b) into the more familiar diagram Fig. 2(a). We will see that the easy-cone regime, characterized by an angle $\theta_{c}$ $=\arcsin \sqrt{\left|K_{1}\right| / K_{2}}$ between the $z$ axis and $\mathbf{n}$, is of particular importance in the present context. It is worth emphasizing that the "leading'" anisotropy constant $K_{1}$ is an effective parameter which may be very small due to demagnetizingfield contributions of order $-\mu_{0} M_{s}^{2} / 2$.,14

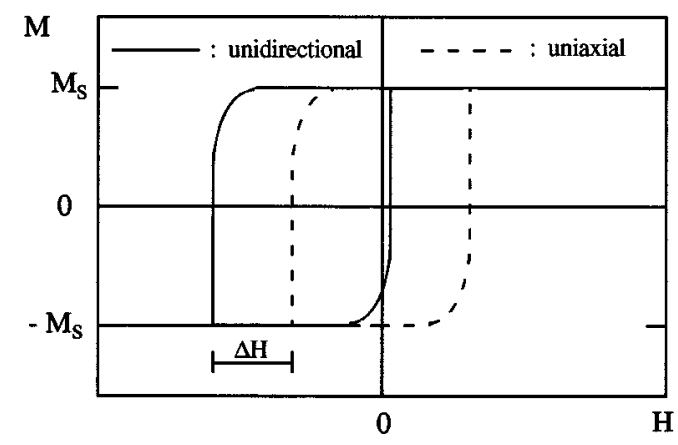

FIG. 1. Uniaxial and unidirectional hysteresis loops (schematic). $M$ is the average magnetization direction parallel to the field $H$. 


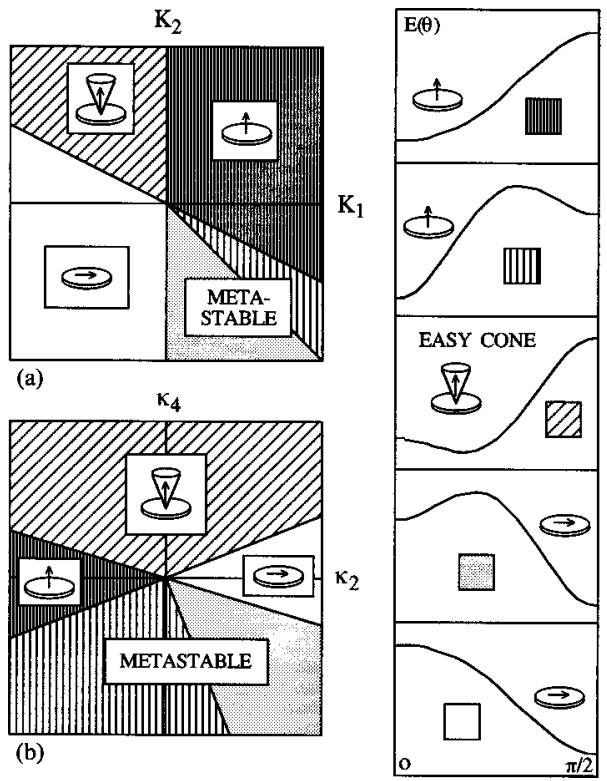

FIG. 2. Basic uniaxial phase diagrams from which the present calculations start: (a) in $K_{1}-K_{2}$ representation and (b) in $\kappa_{2}-\kappa_{4}$ representation. Note that $H=0$ and $E_{a}(\theta)=E_{a}(\pi-\theta)$.

Adding the lowest-order nonuniaxial contributions to Eq. (2) yields the anisotropy energy

$$
\begin{aligned}
E_{a}= & E_{u}+\kappa_{21 c} \cos \theta \sin \theta \cos \phi+\kappa_{21 s} \cos \theta \sin \theta \sin \phi \\
& +\kappa_{22 c} \sin ^{2} \theta \cos 2 \phi+\kappa_{22 s} \sin ^{2} \theta \sin 2 \phi,
\end{aligned}
$$

where $\kappa_{2 m c}$ and $\kappa_{2 m s}$ are nonuniaxial anisotropy coefficients. The $\sin 2 \phi$ and $\cos 2 \phi$ terms are related to the $\cos (2 \phi$ $\left.-2 \phi_{0}\right)$ term in Eq. (2): $2 \phi_{0}=\arctan \left(k_{22 s} / k_{22 c}\right)$ and $K_{1}^{\prime}$ $=\sqrt{k_{22 c}{ }^{2}+k_{22 s}{ }^{2}}$. Here we are interested in the unidirectional coefficients $\kappa_{21 c}$ and $\kappa_{21 s}$, which are ignored not only in general reviews on thin-film and surface anisotropies but also in papers dealing with nonideal surfaces. ${ }^{19}$

From the prefactor $\sin \theta \cos \theta$ we deduce that there is no unidirectional anisotropy for $\theta=0$ and $\theta=\pi / 2$. In the intermediate easy-cone regime, $0<\theta \approx \theta_{c}<\pi / 2$, the magnetization prefers some unique in-plane angle $\phi$. It is important to keep in mind that Eq. (3) does not break the global inversion symmetry $E_{a}(-\mathbf{M})=E_{a}(\mathbf{M})$, which is realized by simultaneously changing $\phi \rightarrow \phi+\pi$ and $\theta \rightarrow \pi-\theta$. In the uniaxial limit, where $E_{a}$ is independent of $\phi$, this symmetry establishes two equivalent cones at $\theta=\theta_{c}$ and $\theta=\pi-\theta_{c}$. However, Fig. 2 shows that the two cones are separated by energy barriers at $\theta=0$ and $\theta=\pi / 2$, so that intercone transitions from $\mathbf{M}$ to $-\mathbf{M}$ require comparatively large activation energies. The external magnetic field necessary to overcome the intercone barrier depends not only on the anisotropy coefficients of the film but also on the field direction. However, in nearly ideal films it is much larger than the unidirectional shift of the hysteresis loop. It is worthwhile noting that a similar energy barrier exists for $\mathrm{Co} / \mathrm{CoO}$-type unidirectional anisotropies. In that case, the field necessary to overcome the barrier is given by the antiferromagnetic $\mathrm{CoO}$ exchange field. Figure 3 shows how the low-symmetry anisotropy contributions contained in Eq. (3) perturb uniaxial energy landscapes. For the practical realization of the energy landscapes Fig. 3 see Sec. III.
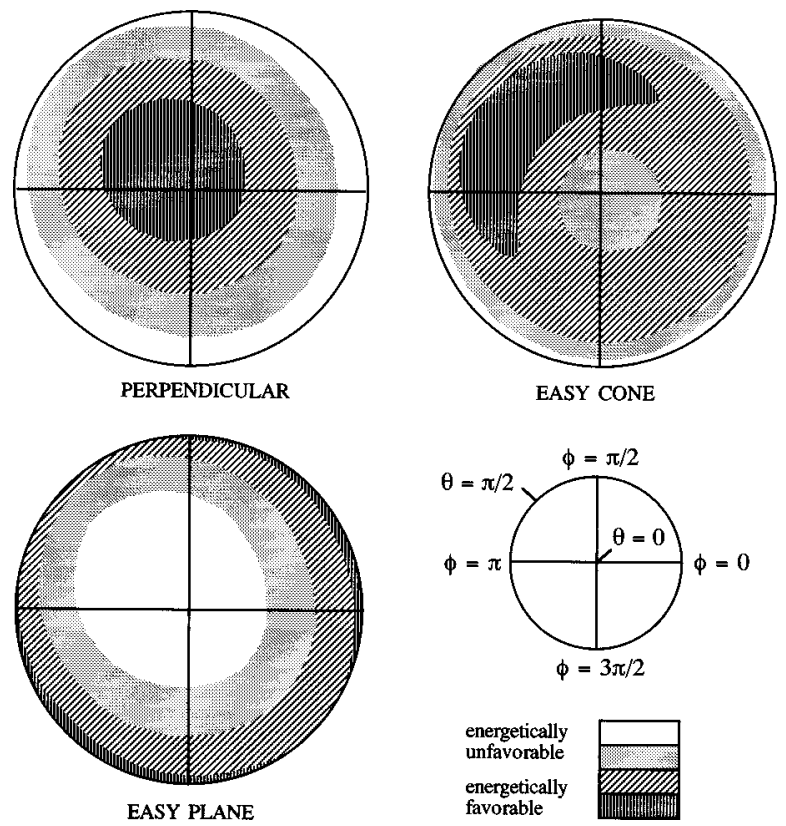

FIG. 3. Typical energy landscapes $E_{a}(\phi, \theta)$ derived from Eq. (3). The terms perpendicular, easy cone and easy axis indicate the uniaxial anisotropy types from which the diagrams derive. The polar axis is $\sin \theta$, and the terms perpendicular, easy cone and easy axis refer to Fig. 2.

To estimate $\kappa_{12 s}$ and $\kappa_{12 c}$ we start from Néel's pair anisotropy energy $g\left(3 \cos ^{2} \alpha-1\right) / 2$, where $\alpha$ is the angle between $\mathbf{n}$ and the real-space vector $\mathbf{r}_{i}-\mathbf{r}_{j}$ connecting the positions of two nearest neighbors, and $g$ is a phenomenological coupling constant. ${ }^{1}$ Note that the applicability of the Néel model to itinerant magnetism is only semiquantitative but gives a good account of the symmetry aspect of the problem. $^{20}$ The spherical harmonic addition theorem ${ }^{21}$ yields, after straightforward calculation, the single-atom coefficients

$$
\begin{gathered}
\kappa_{21 c}=\frac{g}{2} \sum_{i} \sin \Theta_{i} \cos \Theta_{i} \cos \phi_{i}, \\
\kappa_{21 s}=\frac{g}{2} \sum_{i} \sin \Theta_{i} \cos \Theta_{i} \sin \phi_{i},
\end{gathered}
$$

where $\Theta_{i}$ and $\Phi_{i}$ describe the relative position of the $i$ th neighbor. The total anisotropy is obtained by adding all atomic contributions.

\section{III. $\mathrm{Co} / \mathrm{Cu}(11 n)$ SURFACES}

It is interesting to compare the predictions Eq. (4) with the behavior of fcc (11n) surfaces vicinal to fcc (001). ${ }^{11,12,22-24}$ Figure 4 shows the atomic structure of fcc (11n) surfaces, which involve four types of atoms: bulk atoms, surface atoms, step-edge atoms, and step-corner atoms. ${ }^{22}$ Since there are no second-order bulk contributions, we have to deal with $N_{s}$ surface atoms, $N_{c}$ step-corner atoms, and $N_{e}$ step-edge atoms. Up to higher-order terms, the summation procedure yields the CA energy 

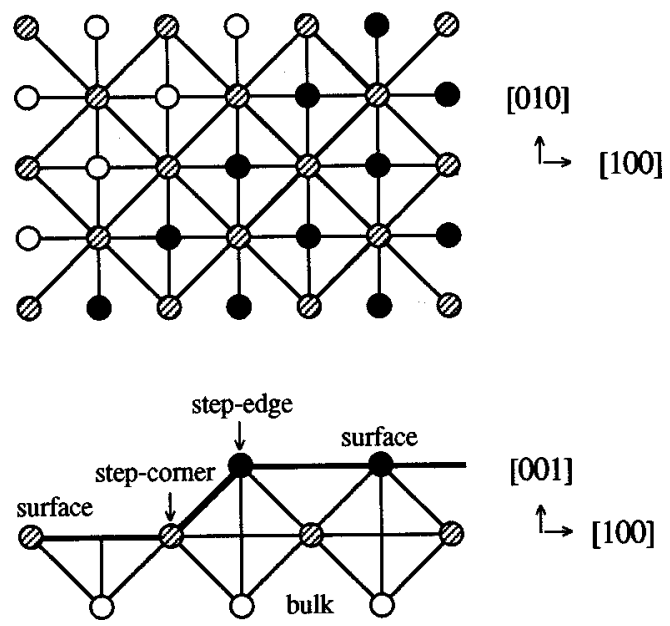

FIG. 4. Morphology of ( $11 n)$ surfaces vicinal to fcc (001). The [100] and [010] directions correspond to $\phi=0$ and $\phi=\pi / 2$ in Fig. 3 , respectively.

$$
\begin{aligned}
E_{a}= & \frac{g}{8}\left(6 N_{\mathrm{eff}}+N_{e} \sin 2 \phi\right) \sin ^{2} \theta \\
& -\frac{g}{2 \sqrt{2}} N_{c} \sin \theta \cos \theta \sin (\phi-\pi / 4),
\end{aligned}
$$

where $N_{\text {eff }}=N_{s}+3 N_{c} / 2+N_{e} / 2$. Since an in-plane external magnetic field applied perpendicular to the step edges yields a Zeeman-energy contribution proportional to $\sin (\phi-\pi / 4)$, the last term in Eq. (5) can be interpreted as a unidirectional anisotropy-field contribution. Independently of the hysteresis mechanism, the fictitious unidirectional anisotropy field $\Delta H$ yields a displacement of the hysteresis loop (Fig. 1). For $g>0$, the preferred magnetization direction is perpendicular to the film plane. In the case of easy-plane anisotropy, $g<0$, the step-edge atoms yield a uniaxial contribution favoring the alignment along the steps. The unidirectional term, which arises from the step-corner atoms, favors the alignment along one of the two in-plane directions perpendicular to the steps. For example, $g<0$ and $\cos \theta>0$ give rise to an easy direction pointing up the steps. This is seen as a shift in the hysteresis loop.

Experiment $^{23}$ shows that the leading $\mathrm{Co} / \mathrm{Cu}(1113)$ anisotropy contribution is easy plane, whereas the steps yield a secondary in-plane easy axis parallel to the direction of the step edges. In terms of Fig. 3, this is the perturbed easyplane limit. The magnitude of the intrinsic unidirectional CA coefficient, $-g N_{c} / 2 \sqrt{2}$ for fcc $(11 n)$ films, is very large, namely, of order $K_{1} / n \approx 0.1 \mathrm{MJ} / \mathrm{m}^{3}$, but this value has to be multiplied by the prefactor $\sin \theta \cos \theta$. This factor is practically zero for the $\mathrm{Co} / \mathrm{Cu}(11 n)$ films, where $\theta \approx \pi / 2$. This indicates that $\kappa_{4}$ is unable to establish the easy-cone regime necessary to make the unidirectional anisotropy visible. Furthermore, the unidirectional shift of the hysteresis loops is most pronounced if the field is parallel rather than perpendicular to the steps. ${ }^{11,12}$

\section{DISCUSSION AND CONCLUSIONS}

A different explanation of unidirectional anisotropies is provided by relativistic Dzyaloshinskii-Moriya (DM) interactions of the type $\mathbf{D}_{i j}\left(\mathbf{S}_{i} \times \mathbf{S}_{j}\right)$, where $\mathbf{D}_{i j}=-\mathbf{D}_{j i}$ determines the anisotropy direction. ${ }^{5,6,8-10}$ RKKY-type spin-glass calculations show that the DM mechanism involves three atoms located at $\mathbf{R}_{0}=0, \mathbf{R}_{i}$, and $\mathbf{R}_{j}$, which determine the direction of the DM vector $\mathbf{D}_{i j} \approx \mathbf{R}_{i} \times \mathbf{R}_{j} .{ }^{8,25}$ In practice, most DM anisotropy contributions cancel each other, but nonzero net contributions occur in low-symmetry magnets. In metallic spin-glasses one assumes that the site $\mathbf{R}_{0}$ is occupied by a nonmagnetic impurity, so that the random distribution of the impurities breaks the symmetry of the lattice. ${ }^{8}$ A similar effect can be expected for stepped surfaces, because surface, step-edge, and step corner atoms have different electronic properties local density of states and moments. From the symmetry of the fcc (11n) surfaces (Fig. 4) follows that the step-corner atoms yield a nonzero DM anisotropy parallel to the step edges, which is in agreement with experiment.

A particular point about the DM anisotropy is that the atomic spins enter the interaction as $\mathbf{S}_{i} \times \mathbf{S}_{j}$, so that they have to be noncollinear to yield unidirectional anisotropy. At this stage it remains open whether this noncollinearity reflects parasitic spin canting as in $\alpha-\mathrm{Fe}_{2} \mathrm{O}_{3}$ (Ref. 10) or micromagnetic deviations from the ideal spin alignment. In any case, we are convinced that this work will stimulate further experimental and theoretical research in the field of unidirectional anisotropies in low-symmetry ultrathin transitionmetal films. This refers not only to the atomic and micromagnetic spin structures but also to problems such as intercone transitions in external fields.

In conclusion, we have established and analyzed the existence of unidirectional anisotropies in stepped ultrathin transition-metal films. In the case of $\mathrm{Co} / \mathrm{Cu}(11 n)$, the unidirectional hysteresis loops are ascribed to DzyaloshinskiiMoriya-type interactions, but in general there is a possibility of a unidirectional anisotropy associated with competing anisotropy coefficients. This anisotropy, which has not been considered in previous work, is nonzero for low-symmetry easy-cone configurations and involves neither DM nor antiferromagnetic exchange.

\section{ACKNOWLEDGMENT}

The authors are grateful to K.-H. Müller for stimulating discussions.

\footnotetext{
*Corresponding author. Present address: Behlen Laboratory of Physics and Center for Materials Research and Analysis, University of Nebraska, Lincoln, NE 68588-0111. Electronic address: rskomski@unlinfo.unl.edu

${ }^{1}$ L. Néel, J. Phys. Radium 15, 225 (1954).

${ }^{2}$ S. Chikazumi, Physics of Magnetism (Wiley, New York, 1964).

${ }^{3}$ W. H. Meiklejohn and C. P. Bean, Phys. Rev. 102, 1413 (1956).
}

${ }^{4}$ N. H. March, P. Lambin, and F. Herman, J. Magn. Magn. Mater. 44, 1 (1984).

${ }^{5}$ I. Dzyaloshinski, J. Phys. Chem. Solids 4, 241 (1958).

${ }^{6}$ T. Moriya, Phys. Rev. 120, 91 (1960).

${ }^{7}$ L. Néel, Ann. Phys. (Leipzig) 2, 61 (1967).

${ }^{8}$ K.-H. Fischer and A. J. Hertz, Spin Glasses (Cambridge University Press, Cambridge, 1991). 
${ }^{9}$ S. Teraoka, T. Kambe, N. Koido, S. Hirai, and K. Nagata, J. Magn. Magn. Mater. 140-144, 1659 (1995).

${ }^{10}$ K. Yosida, Theory of Magnetism (Springer, Berlin, 1996).

${ }^{11}$ W. Wulfhekel, S. Knappmann, B. Gehring, and H. P. Oepen, Phys. Rev. B 50, 16074 (1994).

${ }^{12}$ W. Wulfhekel, S. Knappmann, and H. P. Oepen, J. Appl. Phys. 79, 988 (1996).

${ }^{13}$ A. V. Smirnov and A. M. Bratkovsky, Phys. Rev. B 54, 17371 (1996)

${ }^{14}$ B. Heinrich and J. F. Cochran, Adv. Phys. 42, 523 (1993).

${ }^{15}$ U. Gradmann, in Handbook of Magnetic Materials, edited by K. H. J. Buschow (Elsevier, Amsterdam, 1993), Vol. 7, p. 1.

${ }^{16}$ D. Sander, R. Skomski, C. Schmidthals, A. Enders, and J. Kirschner, Phys. Rev. Lett. 77, 2566 (1996).

${ }^{17}$ P.-A. Lindgård and O. Danielsen, Phys. Rev. B 11, 351 (1975).

${ }^{18}$ Rare-earth Iron Permanent Magnets, edited by J. M. D. Coey
(Oxford University Press, Oxford, 1996).

${ }^{19}$ Note that second-order unidirectional contributions can be eliminated by a unitary transformation, but this "simplification" is paid by the introduction of a fictitious reference frame generally unrelated to directions such as [001] or $[11 n]$. Furthermore, the transformation procedure creates higher-order low-symmetry anisotropy expressions.

${ }^{20}$ See, e.g., R. Skomski, IEEE Trans. Magn. 34, 1207 (1998).

${ }^{21}$ M. T. Hutchings, Solid State Phys. 16, 227 (1964).

${ }^{22}$ D. S. Chuang, C. A. Ballantine, and R. C. O'Handley, Phys. Rev. B 49, 15084 (1994).

${ }^{23}$ A. Berger, U. Linke, and H. P. Oepen, Phys. Rev. Lett. 68, 839 (1992).

${ }^{24}$ P. Krams, B. Hillebrands, G. Güntherodt, and H. P. Oepen, Phys. Rev. B 49, 3633 (1994).

${ }^{25}$ P. M. Levy and A. Fert, Phys. Rev. B 23, 4667 (1981). 